Introduction au thème

\title{
CHERCHEZ LE POLITIQUE... POLYPHONIES, AGENCÉITÉ ET STRATÉGIES DU RAP EN AFRIQUE
}

\author{
Alice Aterianus-Owanga et Sophie Moulard
}

Editions Karthala | « Politique africaine »

$2016 / 1 n^{\circ} 141 \mid$ pages 5 à 25

ISSN 0244-7827

ISBN 9782811116415

Article disponible en ligne à l'adresse :

https://www.cairn.inforevue-politique-africaine-2016-1-page-5.htm

Distribution électronique Cairn.info pour Editions Karthala.

(C) Editions Karthala. Tous droits réservés pour tous pays.

La reproduction ou représentation de cet article, notamment par photocopie, n'est autorisée que dans les limites des conditions générales d'utilisation du site ou, le cas échéant, des conditions générales de la licence souscrite par votre établissement. Toute autre reproduction ou représentation, en tout ou partie, sous quelque forme et de quelque manière que ce soit, est interdite sauf accord préalable et écrit de l'éditeur, en dehors des cas prévus par la législation en vigueur en France. Il est précisé que son stockage dans une base de données est également interdit. 


\title{
LE DOSSIER
}

\author{
Polyphonies du rap \\ Coordonné par Alice Aterianus-Owanga \\ ET SOPHIE MOULARD
}

INTRODUCTION AU THÈME

CHERCHEZ LE POLITIQUE...
POLYPHONIES, AGENCÉtÉ
ET STRATÉGIES DU RAP EN AFRIQUE*

Fin avril 2015, s'ouvrait à l'Institut du monde arabe une exposition intitulée «Hip-hop, du Bronx aux rues arabes ». Réunissant des archives de différentes natures et des créations audiovisuelles conçues pour l'occasion, cet événement mettait en valeur les multiples facettes du hip-hop et de sa discipline orale la plus notoire, le rap ${ }^{1}$. Le projet de l'exposition reposait en grande partie sur l'idée de faire connaître la force de subversion politique de ce mouvement émergeant dans les années 1970 aux États-Unis et propagé tout autour du globe. Le hip-hop y apparaissait comme moyen d'expression «d'un désir grandissant de dignité, de liberté et d'un futur meilleur», et comme "éveilleur de conscience ${ }^{2} »$, notamment dans les États d'Afrique du Nord. Dans cette optique, les dispositifs d'écoute et de visionnage du rap nord-africain mettaient au premier plan des artistes dont les productions musicales avaient été reconnues pour leur dimension subversive, dans les révolutions arabes ou dans la révolte palestinienne contre l'occupation israélienne.

En un sens, cette exposition marqua un tournant dans les institutions culturelles françaises: elle faisait entrer le hip-hop dans un musée de premier

\footnotetext{
* Nous proposons ce titre en clin d'œil à l'article séminal de Denis-Constant Martin à propos du politique et des cultures populaires, auquel font écho certaines réflexions contenues dans ce dossier. D.-C. Martin, «Cherchez le peuple... Culture, populaire et politique», Critique internationale, vol. 7, $\mathrm{n}^{\circ} 1,2000$, p. $169-183$.

1. On reconnaît habituellement quatre disciplines fondamentales au sein du hip-hop: le rap, la danse, le graffiti, le deejaying (art d'enchaîner et de mixer des séquences musicales, souvent à partir de platines vinyles). Le beatboxing (technique vocale d'imitation de sons et d'instruments de musique) s'ajoute parfois à cette liste, de même que la mode et l'élément esthétique.

2. Exposition «Hip-hop, du Bronx aux rues arabes», 28 avril-26 juillet 2015, Paris, Institut du monde arabe, <www.imarabe.org/exposition/hip-hop-du-bronx-aux-rues-arabes>, consulté le 5 avril 2016.
} 
Polyphonies du rap

plan ${ }^{3}$, elle donnait la parole aux acteurs mêmes du mouvement (Akhénaton était le directeur artistique de l'exposition), et elle orientait les regards du public en direction du hip-hop produit de l'autre côté de la Méditerranée, et non plus seulement en France ou aux États-Unis. Parallèlement, en articulant son récit autour de la dimension révolutionnaire du hip-hop, l'exposition perpétuait des visions proposées au cours des dernières années par un ensemble de discours et de publications, qui ont contribué à accroître la visibilité de quelques acteurs du rap africain et les ont institués en hérauts d'une jeunesse africaine contestataire ${ }^{4}$. En effet, quelques mois auparavant, les artistes rap et reggae du Burkina Faso s'étaient fait connaître mondialement en se positionnant en première ligne du mouvement «Balai citoyen» et de l'insurrection d'octobre 2014, qui allait conduire à la transition survenue à l'automne 2015. Plus en amont, les rappeurs sénégalais investis dans le mouvement «Y'en a marre» avaient fait l'objet d'articles et de films documentaires ${ }^{5}$ qui soulignaient leur rôle dans l'alternance politique de 2012.

Ces différents événements ont donné un coup de projecteur médiatique sur un phénomène en plein essor sur le continent africain depuis la fin des années 1980, celui de jeunesses urbaines s'emparant des sonorités hip-hop pour exprimer leurs regards sur leurs sociétés. Implicitement, les publications et l'exposition mentionnées entérinaient une idée communément admise selon laquelle la musique rap et le hip-hop seraient intrinsèquement porteurs d'une fonction de résistance politique et d'engagement contestataire. Le rap, discours de révolte issu des ghettos américains, se serait diffusé et adapté de par le monde tout en conservant une «authentique» visée d'engagement et de dénonciation, trouvant un terreau fertile dans une Afrique en pleine période de transitions démocratiques. Pourtant, par-delà ces images stéréotypées, les positionnements des rappeurs à l'égard du pouvoir se réduisent rarement au registre de la contestation. Ils s'illustrent tout aussi souvent par des positionnements ambigus ou des alliances stratégiques avec le pouvoir, comme l'ont montrées les implications des rappeurs dans les campagnes électorales de plusieurs États, au Gabon, au Kenya ou en Ouganda.

Notre dossier de Politique africaine choisit d'examiner ces relations polyphoniques des rappeurs à l'égard du politique, en se positionnant à rebours

\footnotetext{
3. Plusieurs événements ont témoigné, depuis le milieu des années 2000, de l'entrée du hip-hop dans les institutions culturelles et muséales, notamment l'exposition «Hip-hop, art de rue, art de scène » présentée du 17 juin 2005 au 3 octobre 2005 au Musée des civilisations de l'Europe et de la Méditerranée (Mucem) à Marseille.

4. Voir notamment J.-C. Servant, «L'Afrique conteste en rap», Le Monde diplomatique, 2000, p. 32; Courrier international, «Unis par le rap» numéro spécial, mars 2013, ou encore la revue Africultures, «Culture Hip-hop», n² 21, 1999.

5. Voir notamment A. Gallet, Boy saloum, la révolte des Y'en a marre, France, YAMI 2, 2013, 73 min.
} 
Cherchez le politique... Polyphonies, agencéité et stratégies du rap en Afrique

des présupposés réduisant le rap à son image contestataire et en s'écartant de toute simplification dualiste, que ce soit la dichotomie entre résistance et allégeance, ou celle entre politique par le haut et par le bas. Pour ce faire, le fil conducteur des contributions réunies porte sur les relations changeantes qui allient le rap au politique dans différents contextes historiques et étatiques (Angola, Cameroun, Kenya, Ouganda, Tunisie) et sur l'examen des microstratégies qui permettent aux rappeurs de déployer leur capacité à agir dans la sphère politique.

Pour éclairer ce principe d'hétérogénéité des mondes du rap, nous faisons appel à l'idée de poly phonie. Nous la préférons à d'autres notions bakhtiniennes employées auparavant par des théoriciens du politique en Afrique, telles que celle de dialogisme ou d'énonciation ${ }^{6}$, car elle permet de mettre l'accent sur les contextes et conditions de production, davantage que sur les énoncés eux-mêmes. En outre, l'idée de polyphonie peut s'appliquer au domaine du rap selon deux acceptions distinctes. Une première se rattache à l'approche linguistique qui affirme que tout discours surgit dans un contexte donné, qu'il soit concret ou imaginaire, et que les textes véhiculent des points de vue émanant de différentes sources ${ }^{7}$. Cette première dimension apparaît clairement à l'observation des œuvres des rappeurs (en Afrique et ailleurs) qui mettent en dialogue une série de voix et de regards, au travers de la langue ou des technologies du sampling et de la superposition de séquences sonores. Toutefois, hormis cette évidente dimension linguistique et musicale, nous employons surtout ici la notion de polyphonie selon une seconde acception, plus sociologique. En mettant l'accent sur la voix et non sur l'énoncé luimême ${ }^{8}$, elle invite à considérer les pratiques et processus sous-jacents aux choix et aux discours changeants des individus, plutôt que de s'arrêter à leurs engagements brandis discursivement.

Dans cette perspective, la majorité des contributions de ce dossier favorisent la compréhension des processus au travers desquelles une prise de parole est rendue possible, ainsi que les manières dont elle va se voir attribuer ou non de la valeur politique, plutôt que le contenu textuel lui-même. En corrélation avec nos positionnements théoriques, l'impulsion de ce dossier

6. J.-F. Bayart, «L'énonciation du politique», Revue française de science politique, vol. 35, n 3, 1985 , p. 343-373.

7. M. Bakhtine, La poétique de Dostö̈evski, Paris, Seuil, 1970; O. Ducrot, Le dire et le dit, Paris, Éditions de minuit, 1984; M. Birkelund, H. Nølke et R. Therkelsen, «Introduction. La polyphonie linguistique», Langue française, $\mathrm{n}^{\circ}$ 164, 2009, p. 3-9.

8. À propos de la notion de voix, comme manière processuelle de rendre compte de situations vécues et d'y attribuer ou non de la valeur, voir N. Couldry, Why Voice Matters. Culture and Politics After Neoliberalism, Londres, Sage, 2010. 
Polyphonies du rap

repose donc aussi sur des partis-pris méthodologiques: l'appréhension des détours, les ambiguités et variations du rapport au politique des rappeurs apparaissent dans les cinq articles de ce dossier, par une observation intensive des réseaux musicaux, par une mise en évidence des subdivisions et dissonances en leur sein, et par une entrée sur l'individu plutôt que sur un monde du rap uniformisé. Comme pour le rappeur devenu politicien décrit par Nanna Schneidermann en Ouganda, ou l'artiste kenyan Jaguar évoqué par Daniel Künzler, plusieurs articles retracent des actions individuelles pour comprendre des dynamiques en œuvre dans les régimes politiques concernés.

Par ce prisme, les articles de ce dossier éclairent des écarts entre le «texte public » et le «texte caché» de la vie sociale", et ils interrogent la marge d'agencéité et de réflexivité que s'octroient les individus derrière l'apparent masque de la domination. L'idée d'agencéité (agency) est ici comprise comme la capacité d'agir des acteurs sociaux, incluant ces «marges de manœuvre [...] en situation sociale de semi-contrainte ${ }^{10}$ », mais aussi comme un ressort lié au pouvoir réflexif des individus ${ }^{11}$. Cette dimension réflexive apparaît particulièrement bien dans les discours et entretiens cités par plusieurs contributeurs de ce dossier. Dans le cas de l'Angola décrit par Chloé Buire, cette marge de réflexion et d'expression critique conduit parfois à des "embryons d'élan contre-hégémonique», lorsque se rencontrent les conditions structurelles d'un régime en déclin et des passages à l'action individuels et/ou collectifs.

Ce dossier révèle ainsi la manière dont le rap s'est adapté au sein de différents moments historiques et régimes politiques, accompagnant tantôt leurs déclins, tantôt leurs renouvellements, tantôt leurs reconfigurations. Il souligne aussi les ressorts stratégiques dont les acteurs du monde du rap usent pour négocier une marge d'individuation et d'émancipation dans des espaces verrouillés, au travers de leurs pratiques entrepreneuriales ou de leur capacité à (se) connecter à des réseaux pluriels, à l'échelle locale et transnationale. De la sorte, il éclaire des mécanismes du pouvoir qui avaient, jusqu'à présent, constitué un relatif point aveugle dans les recherches sur le rap en Afrique, bien qu'il résonne avec un plus vaste courant de recherches sur les enjeux politiques des musiques populaires.

9. J. C. Scott, La domination et les arts de la résistance. Fragments du discours subalterne, Paris, Éditions Amsterdam, 2009.

10. J.-P. Olivier de Sardan et T. Bierschenk, «Les courtiers locaux du développement» [en ligne], Bulletin de l'APAD, n 5, 4 juin 2008 [1993], <apad.revues.org/3233>, consulté le 5 mars 2016.

11. S. B. Ortner, Anthropology and Social Theory. Culture, Power and the Acting Subject, Durham, Duke University Press, 2006; R. van Dijk, M. de Bruijn et J.-B. Gewald, «Social and Historical Trajectories of Agency in Africa: an Introduction», in M. E. de Bruijn, R. van Dijk et J.-B. Gewald (dir.), Strength beyond Structure. Social and Historical Trajectories of Agency in Africa, Leyde/Boston, Brill, coll. «African Dynamics», 2007, p. 1-15. 
Cherchez le politique... Polyphonies, agencéité et stratégies du rap en Afrique

\section{DU RAP ET DE SES PRÉdÉCESSEURS: ÉLÉMENTS D'HISTORIOGRAPHIE}

En apparaissant au moment charnière des transitions démocratiques, le rap a fait écho à d'autres périodes où les musiques se sont érigées en support d'expression de pensées politiques ou de mobilisations collectives. En effet, dès les indépendances, les orchestres et les musiciens ont véhiculé des idées sur la nation, la ville et la citoyenneté modernes, dans la Guinée de Sékou Touré$^{12}$, le Mali de Modibo Keïta ${ }^{13}$ ou dans l'ex-Zaïre, où Bob White a montré les liens d'interdépendance entre Mobutu, chef national, et les leaders d'orchestres de rumba ${ }^{14}$. Les orchestres ont constitué le creuset de formation des images de la nation, du panafricanisme ou de l'authenticité, et accompagné la construction d'un imaginaire national ${ }^{15}$ par la musique, la danse et le spectacle ${ }^{16}$.

Au-delà des groupes musicaux étatiques, il est bien connu que les musiciens ont véhiculé des messages puissants de subversion contre des régimes autoritaires, au travers de dénonciations explicites ou de pratiques plus subtiles de la métaphore. En Afrique du Sud, on a vu comment les espaces de musique transgressaient des frontières raciales érigées par l'apartheid, dans les clubs de jazz de Sophiatown notamment, et comment la résistance s'opérait au travers de circulations de musiciens vers l'étranger ${ }^{17}$. Du côté ouest-africain, plusieurs travaux sur le chanteur et saxophoniste Fela Anikulapo Kuti ont insisté sur la dimension révolutionnaire des messages qu'il injecta dans la société nigériane ${ }^{18}$. Bien avant les rappeurs, celui que l'on surnommait le «Black President» apportait déjà un exemple remarquable de «projection

12. C. Pauthier, «La musique guinéenne, vecteur du patrimoine national (des années 1950 à 1984) », in D. Gary-Tounkara et D. Nativel (dir.), L'Afrique des savoirs au sud du Sahara (XVI'e-XXIe siècles): acteurs, supports, pratiques, Paris, Karthala, 2012, p. 129-154.

13. A. Pourrouquet, "La production du patrimoine musical malien», in O. Givre et M. Regnault (dir.), Patrimonialisations croisées. Jeux d'échelles et enjeux de développement, Lyon, Presses universitaires de Lyon, 2015.

14. B. W. White, Rumba Rules: The Politics of Dance Music in Mobutu's Zaire, Durham, Duke University Press, 2008.

15. B. Anderson, L'imaginaire national. Réflexions sur l'origine et l'essor du nationalisme, Paris, La Découverte, 1996.

16. À ce sujet, voir aussi l'ouvrage sur la Tanzanie de K. Askew, Performing the Nation. Swahili Music and Cultural Politics in Tanzania, Chicago/Londres, University of Chicago Press, 2002.

17. Voir notamment D.-C. Martin, «Postface. La musique au-delà de l'apartheid? », in D. Coplan, In Township Tonight, la musique et le théâtre dans les villes noires d'Afrique du Sud, Paris, Karthala, 1996, p. 377-402. À propos de ces circulations de musiciens d'Afrique du Sud, voir aussi R. D. J. Kelley, Africa Speaks, America Answers. Modern Jazz in Revolutionary Times, Cambridge, Harvard University Press, 2012.

18. M. E. Veal, Fela : the Life and Times of an African Musical Icon, Philadelphie, Temple University Press, 2000. 
Polyphonies du rap

de soi comme incarnation d'une culture jeune porteuse de valeurs de sexualité, d'hédonisme et d'antagonisme avec les autorités ${ }^{19}$ ».

Sans se limiter à une perspective opposant des artistes cooptés et des musiciens rebelles, ces travaux sur les musiques populaires africaines ont aussi démontré que les rapports au pouvoir des musiciens ne se reflétaient pas simplement dans les positionnements politiques officiels et les discours publics, et que des micropolitiques plus complexes se manifestaient aussi dans l'organisation sociale des mondes de la musique, les logiques de l'industrie musicale, ou les performances elles-mêmes ${ }^{20}$.

Plutôt que de considérer le rap comme une rupture radicale vis-à-vis de ces précédentes scènes musicales, les contributions réunies dans ce dossier s'attachent à comprendre comment les artistes de hip-hop africain ont interagi avec une plus longue histoire de relations entre musique et politique. Ainsi Chloé Buire, dans son analyse du rap angolais, montre que les scènes de musique populaire contemporaines sont marquées par les décennies préalables de censure et de propagande, autant que par l'incorporation profonde de l'imaginaire national angolais via la musique. De même, Daniel Künzler introduit son étude des stratégies entrepreneuriales des rappeurs kenyans en revenant sur les relations entre musique, média et politique depuis les indépendances, sous Jomo Kenyatta et Daniel Arap Moi. Il montre que bien avant que les rappeurs ne s'affirment comme des «marques" brandies dans le cadre de campagnes politiques, la célébrité de certains musiciens avait déjà pu se poser comme objet de tensions entre les mondes de la musique et du politique.

C'est aussi en observant les scènes hip-hop dans la perspective historique qu'il devient possible de mettre en exergue les singularités du moment où ce genre musical apparaît sur le continent. Dans différents États, l'émergence du rap s'est associée à la période des transitions démocratiques ${ }^{21}$ des années 1980-1990. Cette vague de transformations de régime, survenant apparemment par le «haut», constitua un moment de «passage explicite aux revendications » chez différents groupes ou individus du «bas ${ }^{22} »$. Dans cette

19. J. Labinjoh, «Fela Anikulapo-Kuti. Protest Music and Social Processes in Nigeria», Journal of Black Studies, vol. 13, n 1, 1982, p. 119-134.

20. B. W. White, Rumba Rules..., op. cit.

21. La notion de transition démocratique est ici définie, en accord avec Richard Banégas, comme une "période ambigüe de changement de régime», «de flux institutionnel, d'incertitude, où la norme démocratique tend à s'imposer comme critère de légitimation et oriente les perceptions et les comportements de la majorité des acteurs». R. Banégas, «Les transitions démocratiques: mobilisations collectives et fluidité politique», Cultures $\mathcal{E}$ Conflits, ${ }^{\circ}$ 12, 1993, p. 105-140.

22. J. Siméant, «Protester/mobiliser/ne pas consentir. Sur quelques avatars de la sociologie des mobilisations appliquée au continent africain», Revue internationale de politique comparée, vol. 20, 2013, p. 137. 
Cherchez le politique... Polyphonies, agencéité et stratégies du rap en Afrique

situation de crise, la politique cessa «d'être l'apanage de groupes restreints pour mobiliser des secteurs plus larges de sociétés africaines ${ }^{23} »$, qui purent exercer un rôle et une parole inédite dans l'espace public. Parfois confondue avec un processus de «démocratisation» - qui aboutira souvent à de vives désillusions -, cette période fut surtout source de libéralisation de la scène politique, d'élargissement de l'espace public et de pluralisation des modes d'expression ${ }^{24}$.

À l'instar du zouglou en Côte d'Ivoire ${ }^{25}$, les musiques ont constitué une caisse de résonance puissante de ces transformations des modes d'expression politique dans l'espace public. Dans plusieurs pays où les musiciens précédents devaient user de modes feutrés et de langages métaphoriques pour exprimer leurs critiques, de nouvelles instances nées dans les jeunesses urbaines s'engouffrèrent plus explicitement dans la brèche ouverte par la chute du monopartisme ${ }^{26}$. Des modes inédits d'occupation de l'espace urbain se créèrent autour de la danse, des freestyles, des sound-systems ou des concours de rap, par le canal de cette culture hip-hop importée (dans la plupart des cas) via les circulations des classes aisées. Les jeunes de différentes villes africaines inventèrent alors de nouveaux langages rappés du politique, en s'emparant des techniques vocales observées chez leurs modèles français et américains. Érigés en représentants de la génération des années 1990, ils adoptèrent rapidement les fonctions « d'intellectuels organiques ${ }^{27}$ » que d'autres musiciens portaient bien avant eux ${ }^{28}$, en relayant les idées puisées par le biais de leur consommation de discours et idéologies étrangères, ou de leurs dialogues avec des mouvements locaux.

Ces acteurs en mobilité injectèrent parfois leurs innovations dans les paysages culturels africains à partir de positions interstitielles et d'un certain usage de la dérision, comme l'illustraient encore récemment les représentations

23. J.-P Daloz et P. Quantin (dir.), Transitions démocratiques africaines : dynamiques et contraintes (19901994), Paris, Karthala, 1997, p. 15.

24. Ibid.

25. Y. Konaté, «Génération zouglou» [en ligne], Cahiers d'études africaines, n 168, 25 décembre 2005 [2002], <etudesafricaines.revues.org/166>, consulté le 4 mars 2016.

26. A. Schumann, «Songs of a New Era: Popular Music and Political Expression in the Ivorian Crisis ", African Affairs, vol. 112, n 448, 2013, p. 440-459.

27. A. Gramsci, Textes [en ligne], éd. numérique produite par Jean-Marie Tremblay, Université du Québec à Chicoutimi (Uqac), coll. «Les classiques des sciences sociales», Chicoutimi, 2001 [1983] <classiques.uqac.ca/classiques/gramsci_antonio/textes/gramsci_textes.docl>, consulté le 5 avril 2016.

28. Cette association des rappeurs ou des musiciens populaires aux intellectuels organiques n'est pas spécifique au cas étudié. Voir J. L Decker, «The State of Rap: Time and Place on Hip-Hop Nationalism», Social Text, n³4, 1993, p. 53-84. 
Polyphonies du rap

satiriques du duo de rappeurs ghanéens FOKN Bois ${ }^{29}$. Dans différents cas, c'est aussi par l'ostentation d'esthétiques subversives que se bâtit cette identité hip-hop locale, en jouant sur le port des baggys, bandanas, vêtements amples et bijoux clinquants. Les articles de ce dossier reviennent fréquemment sur le rôle du vestimentaire et du style dans le tournant hip-hop, comme dans le cas angolais où les insignes militaires revêtus par des rappeurs comportent d'éminentes fonctions d'expression d'imaginaires du pouvoir et de la richesse, aux résonances locales et globales.

Par la suite, le hip-hop a continué à accompagner plusieurs mouvements de mobilisation dont il s'est institué en bande-son, dans les Printemps arabes ou dans les processus de transition politique de différents États. Mais il a surtout connu une grande hétérogénéisation interne, allant dans le sens de la diversification des esthétiques et des modes de légitimation. Le cas du Cameroun décrit par Patrick Awondo et Jean-Marcellin Manga offre un exemple évocateur de cette plasticité des scènes rap en fonction des situations historiques et politiques. Au Cameroun, le rap a en effet rapidement été façonné pour exprimer les désirs d'animation, d'hédonisme, de confort matériel ou de sexualité, s'affirmant comme le support d'expression des imaginaires de la jeunesse. Au-delà de cet exemple, dans différents États du continent, les secondes générations de rappeurs adaptèrent souvent le rap dans le sens de l'animation populaire, en lien avec des objectifs de commercialisation pérenne de leurs productions. Pour s'insérer de façon durable dans les industries musicales locales, ils allièrent les sonorités hip-hop en vogue aux États-Unis, telles que le dirty south ou la trap, avec les rythmiques dansantes inspirées du ndombolo, plus favorables dans les scènes musicales locales.

Embryonnaires dans les années 1980, et encore marginales dans les années 1990, les musiques hip-hop occupent depuis les années 2000 une place prépondérante dans les paysages musicaux et les ambiances urbaines africaines. Les réseaux constitués autour des genres hybrides nés de la rencontre entre les styles musicaux locaux et le hip-hop (kwaïto, zouglou, kuduro, hiplife, naija music, etc.) se sont structurés et ont conduit, dans plusieurs pays, à la création de véritables économies musicales, inondant les industries nationales (au Sénégal par exemple) ou continentales (pour le cas des musiques nigérianes et sud-africaines). Le développement des technologies du numérique et la dématérialisation des œuvres ont concouru à de nouveaux modes de consommation, de réception et de circulation de ces sonorités et à une influence croissante des acteurs du hip-hop dans l'espace public.

29. «FOKN Bois - BRKN LNGWJZ (Broken Languages)», YouTube, 11 décembre 2011, <www. youtube.com/watch?v=wdg-_TRiNkw>, consulté le 5 avril 2016. Les FOKN Bois sont un duo de rappeurs connus au Ghana pour leur style parodique et irrévérencieux. 
Cherchez le politique... Polyphonies, agencéité et stratégies du rap en Afrique

Les contributions réunies dans ce dossier s'intéressent à ces contours changeants des scènes rap africaines et à leurs articulations avec les moments historiques qu'elles ont accompagnés. En traitant de contextes et au travers d'approches conceptuelles fort différentes, elles possèdent pour ligne commune de s'éloigner de la quête d'une signification politique intrinsèque des pratiques des rappeurs. En cela, notre dossier réagit vis-à-vis d'un champ d'études qui se développe à propos du hip-hop en Afrique depuis le début des années 2000 et qui porte un intérêt croissant à la question politique.

\section{Des rap studies en Afrique}

En parallèle de l'expansion des musiques hip-hop sur le continent africain, les chercheurs en sciences sociales n'ont pas manqué de porter leur attention sur ces phénomènes culturels urbains. Comme dans d'autres espaces d'implantation, le rap a d'abord été appréhendé au travers d'études des textes et des contenus langagiers ${ }^{30}$, par exemple par des descriptions des variations linguistiques des jeunes du Gabon, du Sénégal ${ }^{31}$ ou d'Afrique de l'Est $^{32}$. Ces premiers travaux ont essentiellement appréhendé le rap comme un énoncé discursif, plutôt que comme un creuset de pratiques sociales et culturelles, sans nécessairement envisager le discours lui-même comme une pratique sociale $^{33}$. Le politique y apparaissait comme l'une des thématiques des textes de rap, liée aux codes subversifs et provocateurs du genre. Mais par-delà les mots, le rapport au politique des artistes n'était pas mis au centre des descriptions, ni problématisé en tant que tel, hormis dans quelques travaux ${ }^{34}$.

Comment le rap avait-il fait sens dans ces situations politiques particulières? Par quels mécanismes les jeunes Africains étaient-ils parvenus à en faire un

\footnotetext{
30. Les ethnographies consacrées au rap du continent africain émergent plus tardivement en France qu'aux États-Unis (où existait déjà un champ solide de rap studies), malgré quelques travaux pionniers au début des années 2000. Voir M. Auzanneau, «Identités africaines: le rap comme lieu d'expression», Cahiers d'Études africaines, n 163-164, 2001, p. 711-734; H. Miliani, «Culture planétaire et identités frontalières », Cahiers d'Études africaines, n 168, 2002, p. 763-776.

31. M. Auzanneau, M. Bento et V. Fayolle, «De la diversité lexicale dans le rap au Gabon et au Sénégal», La linguistique, vol. 1, n 38, 2002, p. 69-98.

32. A. Perullo et J. Fenn, «Language Ideologies, Choices, and Practices in Eastern African Hip Hop », in H. M. Berger et M. T. Carroll (dir.), Global Pop, Local Language, University of Mississipi Press, 2003, p. 19-51.

33. En termes de thématiques abordées, ce sont avant tout les constructions identitaires et les logiques d'appropriations localisées du hip-hop en contexte mondialisé qui ont retenu l'attention de ces premières recherches. Voir, entre autres, R. Ssewakiryanga, «Imaginer le monde chez soi. Les jeunes et la musique internationale en Ouganda», Politique africaine, n 75, 1999, p. 91-106.

34. J.-F. Havard, «Ethos "bul faale" et nouvelles figures de la réussite au Sénégal», Politique africaine, $\mathrm{n}^{\circ} 82$, p. 63-77.
} 
Polyphonies du rap

outil d'action sur leurs contextes sociaux? Quelles accommodations aux logiques locales de relation entre musique et politique avait-il rencontrées? Et à l'inverse quelles nouvelles formes de dialogues avec le pouvoir avait-il engendrées?

Ces questions commencèrent à éveiller l'attention des chercheurs dans la suite du développement des études sur le rap en Afrique. Progressivement, un lot de travaux a mis à profit d'autres entrées méthodologiques, par des approches ethnographiques de longue durée, concourant à l'édification d'une historiographie plus précise des modalités d'adoption du hip-hop sur le continent ${ }^{35}$. On vit alors se développer d'autres traitements de la question politique, révélant notamment comment les enjeux de pouvoir transparaissaient de façon feutrée et par le truchement d'autres champs du social, dans le registre du religieux par exemple ${ }^{36}$. Dans le domaine des rapports de genre également, plusieurs travaux ont souligné les enjeux politiques contenus dans l'affirmation de la masculinité et la reproduction d'idéologies conservatrices des rappeurs ${ }^{37}$. D'autres études ont éclairé l'équilibre complexe entre la remise en question d'inégalités ou de stéréotypes de genre et la reproduction de systèmes de contrainte et de mécanismes de domination masculine ${ }^{38}$. Parallèlement, la compréhension du positionnement marginal ou «liminaire ${ }^{39}$ » des rappeurs dans le champ social a représenté un aspect important de la réflexion sur le pouvoir dans le rap $^{40}$, en lien avec les discussions sur l'idée de «jeunesse» et sur l'histoire des luttes politiques dont elle a pu être le moteur ${ }^{41}$.

35. A. Perullo, «"Here's a Little Something Local” : an Early History of Hip Hop in Dar es Salaam, Tanzania, 1984-1997», in J. R. Brennan, A. Burton et Y. Qwaray Lawi (dir.), Dar Es Salaam: Histories from an Emerging African Metropolis, Dar Es Salaam/Nairobi, Mkuki Na Nyota Publishers/The British Institute of Eastern Africa, 2007, p. 250-272; E. Charry, «Introduction: a Capsule History of African Rap», in E. Charry (dir.), Hip-Hop Africa. New African Music in a Globalizing World, Bloomington, Indiana University Press, 2012, p. 1- 25.

36. A. Niang, «Le rap prédicateur islamique au Sénégal: une musique "missionnaire" », Volume!, vol. 10, n², 2014, p. 69-86.

37. E. Mwangi, «Masculinity and Nationalism in East African Hip-Hop Music», Tydskrif Vir Letterkunde, vol. 2, n 41, 2004, p. 5-20.

38. J. Shipley, Living the Hiplife. Celebrity and Entrepreneurship in Ghanaian Popular Music, Durham, Duke University Press, 2013; A. Aterianus-Owanga. " "L'émergence n'aime pas les femmes!" Hétérosexisme, rumeurs et imaginaires du pouvoir dans le rap gabonais », Politique africaine, $\mathrm{n}^{\circ} 126$, 2012, p. 49-68.

39. S. Moulard-Kouka, "Senegal yewuleen!" Analyse anthropologique du rap à Dakar: Liminarité, contestation et culture populaire, thèse de doctorat en anthropologie, Bordeaux, Université de Bordeaux 2, 2008.

40. A. Perullo, «Hooligans and Heroes: Youth Identity and Hip-Hop in Dar Es Salaam, Tanzania», Africa Today, vol. 51, n 4, 2005, p. 75-101.

41. F. De Boeck et A. Honwana (dir.), Makers and Breakers. Children and Youth in Postcolonial Africa, Oxford/Dakar/Trenton, James Currey/Codesria/Africa World Press, 2005. 
Concernant la question du politique, deux schémas interprétatifs dominants semblent s'opposer dans cette littérature sur le rap en Afrique. Majoritaire, un premier type de discours présente le rap comme essentiellement voué à critiquer les maux de la société, porte-voix des classes déshéritées et doté d'une fonction contestatrice garante de son «authenticité». Cette vision romantique envisage les rappeurs comme inventeurs de systèmes symboliques alternatifs et porteurs d'une force de subversion des régimes dominants. La seconde interprétation, apparemment antagonique, présente ces artistes comme aliénés par un ordre politique et culturel hégémonique, que ce soit celui du capitalisme néolibéral ou des figures dominantes locales.

En réalité, ces deux approches se conjuguent souvent dans des écrits qui condamnent à demi-mot les musiques hip-hop aux accents festifs et hédonistes ( $\mathrm{R}^{\prime} \mathrm{n}^{\prime} \mathrm{B}$, bongo flava, ntcham), au profit d'autres artistes qui auraient continué à porter bien haut les couleurs d'un rap critique «authentique». Se basant sur le binarisme entre hip-hop mainstream et hip-hop underground souvent érigé par les artistes eux-mêmes, Msia K. Clark défend par exemple, dans plusieurs ouvrages, l'idée d'une origine du hip-hop dans la contestation, affirmant que l'Afrique serait aujourd'hui un «espace où réside le hip-hop "authentique" 42 ». Dans l'ouvrage collectif qu'elle dirige ${ }^{43}$, plusieurs contributions reprennent ainsi l'idée du « retour aux origines » que représenterait l'adoption du rap en Afrique, un art «entièrement indépendant des marchés dominants » prônant la solidarité et l'unité 44 .

Au-delà du continent africain, cette vision dualiste a imprégné les premières études américaines qui s'étaient emparées de la culture hip-hop aux ÉtatsUnis et qui la voyaient comme $l^{\prime}$ «authentique sensibilité du ghetto ${ }^{45}$ » recherchée pour comprendre les cultures afro-américaines ${ }^{46}$. Loin d'être singulière au mouvement hip-hop, cette division du rap se rattache aussi à

42. M. K. Clark, «Representing Africa! Trends in Contemporary African Hip Hop », Journal of PanAfrican studies, vol. 6, n³, 2013, p. 3.

43. M. K. Clark et M. K. Mwanzia (dir.), Hip Hop and Social Change in Africa: Ni Wakati, Lanham, Lexington Books, 2014.

44. Dans l'ouvrage collectif qu'il éditait sur le hip-hop africain, Paul Khalil Saucier soulignait cette tendance en parlant d'une difficulté à aborder le phénomène hip-hop sans verser dans l'opposition entre contre-hégémonie et consommation capitaliste. P. Khalil Saucier (dir.), Native Tongues. An African Hip-Hop Reader, Trenton, Africa World Press, 2011, p. XIX.

45. R. D. J. Kelley, «Looking for the "Real" Nigga: Social Scientists Construct the Ghetto", in M. Forman et M.A. Neal (dir.), That's the Joint! The Hip-hop Studies Reader, New York, Routledge, 2011 [1997], p. 134-152.

46. Dans une tendance proche, le rap a aussi été assimilé en France par les médias et les premières publications sur le hip-hop à un univers, objet de constructions médiatiques exotisantes, celui de la «banlieue». Pour des critiques à ce propos, voir A. Pecqueux, Voix du rap. Essai de sociologie de l'action musicale, Paris, L'Harmattan, 2007; K. Hammou, Une histoire du rap en France, Paris, la Découverte, 2012. 
Polyphonies du rap

une tendance plus globale caractérisant l'interprétation des «cultures populaires », bien connue des sociologues de la culture. Dans un ouvrage classique, Claude Grignon et Jean-Claude Passeron ${ }^{47}$ analysaient déjà les contours de ces lectures du populaire, en parlant d'une oscillation entre un «populisme» qui envisage les cultures populaires comme des systèmes symboliques autonomes et une approche «misérabiliste», qui réduit ces cultures à des reproductions du regard dominant. Différents sociologues, y compris ceux travaillant sur le rap français ${ }^{48}$, ont appelé à dépasser cette césure en considérant le rapport au politique comme pratique culturelle et comme expérience sociale ${ }^{49}$.

Dans leur prolongement, plusieurs recherches récentes sur le rap en Afrique ont tenté de résoudre cette équation et d'améliorer la compréhension de la dialectique entre résistance et domination, en commençant par remettre en question l'idée que le rap posséderait des fonctions inhérentes de contestation sociale. Certaines études ont fourni des éclairages nouveaux sur ces questions en documentant des situations d'implication des rappeurs dans les processus électoraux. En Tanzanie, Uta Reuster-Jahn a décrit comment, lors de l'élection de 2005, des chansons de bongo flava relevaient tantôt de la protestation, de la moquerie ou de la louange et avaient offert aux artistes l'opportunité d'élargir le spectre de leurs audiences ${ }^{50}$. La montée en puissance du bongo flava a alors accompagné l'émergence de nouvelles personnalités charismatiques dans les sphères décisionnaires ${ }^{51}$. L'impact des chansons de rap a aussi été discuté au Kenya, notamment à travers l'exemple de la chanson «Unbwogable» de Gidi Gidi Maji Maji ${ }^{52}$, qui «sans être explicitement associée à tel ou tel parti politique», exprimait la volonté de renversement du régime de Daniel Arap Moi en 2005, avant d'être reprise par des groupes d'opposition. Dans ces travaux, les productions musicales étaient appréhendées comme des objets en circulation, employées de façon stratégique par les rappeurs et leurs audiences, et dont il s'agissait de retracer les processus d'attribution de la valeur politique.

47. C. Grignon et J.-C. Passeron, Le savant et le populaire. Misérabilisme et populisme en sociologie et en littérature, Paris, Seuil, 1989.

48. A. Pecqueux, Voix du rap..., op. cit.

49. Ibid., p. 10. À propos des liens entre culture populaire et politique, voir les travaux de D.-C. Martin, «Cherchez le peuple...», art. cité; J.-R. Bennetta et D.-C. Martin, Cultures populaires, identités et politique, Les Cahiers du CERI, n 17, 1997.

50. U. Reuster-Jahn, "Bongo Flava and the Electoral Campaign 2005 in Tanzania», Stichproben. Wiener Zeitschrift für kritische Afrikastudien, vol. 14, n 8, 2008, p. 41-69.

51. B. Englert, «Ambiguous Relationships: Youth, Popular Music and Politics in Contemporary Tanzania", Stichproben. Wiener Zeitschrift für kritische Afrikastudien, vol. 14, n 8, 2008, p. 71-96.

52. W. Bender, «Le hip-hop au Kenya: créateur d'identité ou nouvelle "musique nationale" ? L'exemple d"“Unbwogable” de GidiGidiMajiMaji», Cahiers d'ethnomusicologie, n² 20, 2007, p. 107-131. 
Dans le même temps, la connaissance des rapports au politique s'est aussi améliorée par des descriptions des coulisses des participations politiques des rappeurs, au-delà de leurs chansons. Lélection présidentielle de 2009 au Gabon, théâtre d'une scission de la scène rap, a apporté un exemple évocateur à ce propos, révélant comment ces implications relevaient de processus de négociations entre musiciens et politiciens et d'une instrumentalisation mutuelle. La performance rappée d'Ali Bongo lors d'un spectacle de campagne révéla à l'époque que les acteurs politiques remodelaient leurs représentations en incluant les éléments de la culture hip-hop, en vue d'acquérir une légitimité symbolique à l'égard de la jeunesse ${ }^{53}$. L'article de Nanna Schneidermann dans ce dossier poursuit cette réflexion en décrivant, lui aussi, un président transfiguré en rappeur, en Ouganda cette fois-ci. Il relate comment, durant la campagne électorale de 2011, le président Museveni fit paraître un morceau mêlant le rap aux registres traditionnels des comptines locales, qui connut un succès spectaculaire dans les médias. En Ouganda comme au Gabon, les présidents empruntent désormais les codes imposés par la culture hip-hop pour acquérir davantage de capital symbolique, tandis que les rappeurs emploient leur notoriété pour se rapprocher des postes de pouvoir. Dans ces deux États, les performances rappées des candidats-présidents résultaient de collaborations entre politiciens et musiciens, et elles rendaient preuve des modalités inédites de relations du politique aux musiciens induites par l'impact du hip-hop dans l'espace public.

Plus récemment enfin, certains travaux ont rappelé combien les domaines apparemment en dehors du politique étaient eux aussi porteurs d'une forte capacité critique dans le hip-hop. Thomas Fouquet a ainsi traité des «politiques du style ${ }^{54}$ » observées chez plusieurs figures dakaroises, en prolongeant les remarques de Christian Béthune sur le pouvoir critique des formes d'expression mineure ${ }^{55}$. Il confirmait ainsi l'importance de chercher le politique au-delà de ses champs d'expression «majeurs», du côté du culturel et du style lui-même.

Dans la continuité de ces travaux, nous suggérons nous aussi, dans ce dossier, que le dépassement des biais subjectifs accompagnant la lecture du rap nécessite de s'extraire des lectures du politique comme seule contestation frontale, engagement formel ou enrôlement explicite. À l'inverse, une partie

53. A. Aterianus-Owanga, «Rap et démocratie dans le Gabon contemporain. Les stratégies musicales d'invention du politique», Émulations, n 9, 2011, p. 43-55.

54. T. Fouquet, «La trame politique des cultures urbaines: motifs dakarois», in K. Tall, M.-E. Pommerolle et M. Cahen (dir.), Collective Mobilisations in Africa. Enough is Enough!/Mobilisations collectives en Afrique. Ça suffit!, Leyde, Brill/Africa-Europe Group for Interdisciplinary Studies, 2015, p. 112-141.

55. C. Béthune, «Le hip-hop: une expression mineure», Volume!, vol. 8, n² 2, 2011, p. 161-185. 
Polyphonies du rap

des contributions de ce dossier éclairent les mécanismes subtils et variés au travers desquels les scènes rap s'encastrent dans des ordres hégémoniques et participent de leur dynamique.

FAÇONNER LA VOIX DU RAP: HÉGÉMONIE, DOMINATION ET CONSENTEMENT

Inscrites dans une longue tradition de sociologie du politique, les notions d'hégémonie et de domination ont été mobilisées dans des études portant sur des contextes européens et américains, avant d'être revisitées par les auteurs s'attachant à des aires culturelles extra-occidentales, en Inde, puis en Afrique. Bon nombre d'auteurs, dans le champ des études africaines, se sont emparés de la notion gramscienne d'hégémonie idéologique ou culturelle afin de dépasser la logique de la violence étatique et de comprendre comment l'adhésion de la population aux valeurs de l'élite dominante s'assoit sur d'autres champs sociaux ou culturels. Dans ce prolongement, Béatrice Hibou a montré comment le maintien de l'État dans la Tunisie de Ben Ali passait autant par la coercition que par la recherche de légitimité, la fabrique du consentement, voire la séduction ${ }^{56}$. C'est ainsi que l'État assure le fonctionnement du binôme commandement-obéissance et ancre son pouvoir sur le socle d'une certaine légitimité. Dans ce registre d'idées, les institutions culturelles et les musiques populaires ont exercé un rôle nodal en Afrique, celui de fabrication d'un imaginaire national et de représentation du corps du pouvoir.

Comme le montrent plusieurs articles de ce dossier, le rap en Afrique s'est, pour partie, inséré dans ces appareils d'État de régimes politiques autoritaires ou semi-autoritaires. Patrick Awondo et Jean-Marcellin Manga apportent des éléments de discussion à ce propos en traitant d'une scène rap dont l'influence et l'impact politique sont restés, en apparence, bien moins revendicatifs que dans d'autres États, celle du Cameroun. Leur contribution montre comment les contenus de la parole rap ont été en partie façonnés par des discours locaux exogènes (des médias, des auditeurs, des amateurs), par les configurations de l'industrie musicale existante et par un régime politique certes vieillissant, mais toujours verrouillé. À l'inverse d'États où il a sonné le glas des régimes autoritaires, le rap a été confronté au Cameroun à une industrie musicale foisonnante, où la fonction de dénonciation politique était déjà portée par plusieurs figures emblématiques. À défaut de se construire comme genre musical engagé, il a revêtu les fonctions d'animation pour négocier avec un

56. B. Hibou, Anatomie politique de la domination, Paris, La Découverte, 2011. 
régime qui «[...] sait, par une propagande minutieusement orchestrée, décrédibiliser des acteurs portant une parole critique» et dont les techniques de contrôle innervent la scène rap, comme le suggèrent Patrick Awondo et Jean-Marcellin Manga (infra).

L'article de Stefano Barone et d'Elham Golpushnezad prolonge les réflexions sur la gestion par les rappeurs de contextes semi-autoritaires, en décrivant l'évolution de la scène hip-hop tunisienne dans la période post-révolutionnaire. Contrairement à l'idée d'une rupture des mécanismes de domination politique qu'aurait accompagnée le rap après la Révolution de 2011, les auteurs éclairent l'existence d'une continuité dans l'exercice de la domination, la fabrique du consentement, et les rapports entre rap et politique. La discipline s'exerce d'un côté grâce à l'inclusion des artistes dans l'appareil bureaucratique et les activités étatiques (festivals, concerts publics) et de l'autre, par la répression des activités survenant en dehors du système. À nouveau, des débats de légitimité et des conflits naissent autour des compromis que développent les rappeurs entre contestation et dissidence, induisant une déstabilisation de la scène locale.

En Tunisie, en Ouganda ou au Cameroun, ces conflits internes aux scènes rap peuvent être considérés comme d'éminents révélateurs de l'hétérogénéité des réseaux musicaux, brisant l'image monolithique qui en est trop souvent donnée. Ils reflètent des polémiques internes au milieu hip-hop concernant le rôle de l'artiste rap ou du musicien «authentique», aussi bien que des lignes de tensions qui caractérisent plus largement le rapport au politique dans la société.

En abordant les contours de la déqualification politique du rap au Cameroun ou les dispositifs d'inclusion des rappeurs au sein du pouvoir en Tunisie, ces articles soulignent par conséquent l'importance de considérer le rap non seulement en lien avec le contexte politique étatique avec lequel il interagit, mais aussi en relation avec les industries culturelles et musicales existantes où il est venu prendre position et dont il a en partie adopté les normes, au niveau local et global. La qualification du rap comme expression politique ou apparemment apolitique s'opère par le biais d'acteurs et de discours extérieurs, ceux des médias, des auditeurs, des rumeurs et des consommateurs. En ce sens, dans le dialogue entre les acteurs des mondes du rap, celui-ci devient bien une arène de discussions sur le pouvoir.

Parallèlement, face à ces situations d'enchâssement des artistes hip-hop dans les rouages de pouvoirs hégémoniques, se pose la question des moyens de négociation d'un espace d'expression, d'émancipation et de réflexivité par les acteurs sociaux. Notre dossier démontre que l'existence de puissants cadres de contrainte n'anéantit pas la possibilité d'une expression critique ou d'une action sur les hiérarchies sociales par les rappeurs. 
Polyphonies du rap

\section{CÉlÉBRITÉ, ENTREPRENEURIAT ET STRATÉGIES NÉOLIBÉRALES}

Plusieurs articles de ce dossier exposent la façon dont, en vue de s'autonomiser des cadres de domination, les rappeurs jouent de leur célébrité dans une dynamique entrepreneuriale. Daniel Künzler décrit par exemple les rappeurs du Kenya au travers de la figure d'«entrepreneur de lui-même», notion qu'il mobilise en vue de dépasser l'analyse dualiste du rap (comme expression contestataire ou comme manifestation d'une «intimité avec la tyrannie ${ }^{57}$ »). Inspiré de Foucault, ce concept-clé lui permet d'éclairer les relations versatiles des rappeurs kenyans à l'égard du pouvoir institutionnel, qui s'organisent autour de plusieurs stratégies telles que le fait de construire une marque autour de sa notoriété, d'adopter des positions versatiles ou d'ouvrir de nouveaux marchés de collaboration. Il affirme que la compréhension de ces stratégies est indissociable de celle des pratiques néolibérales de l'État kenyan contemporain, qui imprègne les relations au politique des rappeurs: ceux-ci vendent leurs œuvres au plus offrant et s'adaptent aux opportunités du marché, en tâchant de se prémunir de tout engagement formel.

Le cas du rap kenyan, tout comme les remarques émises auparavant dans les travaux sur l'entrepreneuriat dans le hip-hop sénégalais ${ }^{58}$ ou ghanéen ${ }^{59}$, confirme que le rapport des jeunesses africaines contemporaines au pouvoir s'articule autour d'une certaine appropriation de la pensée néolibérale. Il prouve que des citoyennetés et agencéités néolibérales plurielles se bâtissent ${ }^{60}$, en des lieux où le néolibéralisme n'est pas seulement synonyme de soumission à d'implacables logiques de marchés aliénantes, mais aussi de nouveaux ressorts d'individuation. Ainsi dans le contexte ougandais, des rappeurs se produisent dans les concerts de campagne électorale, considérant cette prestation comme d'ordre professionnel, mais ne s'en réservent pas moins (d'après leurs déclarations), la liberté de voter pour qui bon leur semble le jour du scrutin. De même, un rappeur tunisien cité par Elham Golpushnezad et Stefano Barone reconnaît produire des chansons qui ne reflètent pas ses propres convictions, en réponse aux commandes des bailleurs. L'ethnographie intensive permet ici de distinguer les discours

57. L'auteur reprend à ce propos les travaux d'Achille Mbembe, De la postcolonie. Essai sur l'imagination politique dans l'Afrique contemporaine, Paris, Karthala, 2000.

58. J. F. Mbaye, Reconsidering Cultural Entrepreneurship: Hip-Hop Music Economy and Social Change in Senegal, Francophone West Africa, thèse de doctorat en philosophie, Londres, London School of Economics and Political Science, 2011.

59. J. Shipley, Living the Hiplife..., op. cit.

60. A. Ong, «Les mutations de la citoyenneté», Rue Descartes, n 67, 2010, p. 109-117. 
publics des discours privés, et de démontrer que ce qui s'apparente à une dissonance peut être également compris comme une stratégie d'individuation, par un cloisonnement des sphères du public et de l'intime.

Comme le défendent plusieurs articles du dossier, cette démarche entrepreneuriale conduit parfois certains acteurs du milieu hip-hop à faire levier sur les hiérarchies sociales, sur le plan individuel ou collectif. L'article de Nanna Schneidermann sur le rap ougandais offre un exemple très riche de ces manières de se frayer un passage et une marge de réussite dans les ordres de pouvoir par des stratégies entrepreneuriales. Avec l'exemple du producteur et musicien Eddy Yawe et de sa participation aux élections parlementaires de 2011, Nanna Schneidermann montre comment les réseaux d'influence acquis dans les milieux de musique et de la production hip-hop parviennent parfois à s'étendre bien au-delà de l'industrie musicale, jusqu'aux arènes du pouvoir. Pour se hisser dans les sphères de décision politique, les artistes hip-hop font alors usage de leur popularité auprès des classes populaires et de leurs fonctions de médiateurs avec la jeunesse ou le «ghetto ».

Au-delà des cas kenyans et ougandais, cette figure de l'entrepreneur culturel, médiateur connectant les réseaux et s'élevant par ce biais dans les hiérarchies locales, s'applique à bien d'autres rappeurs africains qui se rapprochent des sphères politiques, particulièrement au sein des premières générations de rappeurs. Didier Awadi, au Sénégal, s'impliqua notamment dans l'organisation nationale du Festival mondial des arts nègres (Fesman) de 2010 et dans des projets de la ville de Dakar, ce qui ne l'empêcha pas de rejoindre par la suite le vent de l'alternance. Au Gabon, plusieurs rappeurs et producteurs accédèrent à des postes de conseillers à la présidence suite aux élections de 2009 et d'autres jouent, aujourd'hui encore, de leur emploi au ministère de la Culture pour étendre leurs réseaux d'influence dans la sphère politique. Bien que leurs trajectoires diffèrent, ces rappeurs se rejoignent par le fait d'avoir créé des entreprises autour de leur activité musicale et de concevoir leurs actions politiques selon des intérêts marchands. Ils jouent de façon ouverte de leur faculté à relier différentes sphères et réseaux, ce qui ne les empêche pas, le moment venu, de faire valoir des convictions plus affichées pour entrer en politique.

C'est par des intrications entre réseaux politiques et musicaux que les rappeurs s'engagent dans ces transactions de pouvoir ${ }^{61}$. Celles-ci sont parfois

61. Comme Nanna Schneidermann dans ce dossier, plusieurs chercheurs travaillant sur le rap font, pour cette raison, appel à l'analyse des réseaux. Voir aussi à ce sujet K. Hammou, «Artistes, professionnels, stars. L'histoire du rap français au prisme d'une analyse de réseaux», in B. JoyeuxPrunel et L. Sigalo Santos (dir.), L'art et la mesure. Histoire de l'art et méthodes quantitatives, Paris, Rue d'Ulm, 2010, p. 331-346. 
Polyphonies du rap

le lieu d'une émancipation et d'une ascension sociale, bien qu'elles restent imprévisibles et potentiellement réversibles, lorsque certaines limites érigées par les discours d'authenticité des rappeurs sont franchies. Ainsi, une autre création réunissant les plus grands noms de la scène musicale ougandaise autour du président Museveni a rencontré un échec notoire. Pour Nanna Schneidermann, le fait que ces artistes soient passés des rôles de «courtiers culturels», «connectant» les hommes politiques et les classes populaires, aux rôles de «clients», explique l'échec de ce projet: la légitimité repose ici, pour les artistes hip-hop, sur le fait de «ne pas s'impliquer dans des relations de patronage classique, mais plutôt de garder la nature de leur engagement ouvert à l'interprétation ». Ainsi, cet exemple rappelle que les stratégies entrepreneuriales des artistes sont certes liées aux idéologies néolibérales des régimes en question, mais que celles-ci sont aussi contrebalancées par des enjeux de légitimité clivant les scènes rap.

\section{TACTIQUES DE L'ESPACE ET GÉOGRAPHIES CONTRE-HÉGÉMONIQUES}

Une autre clé de compréhension des modes de négociation de l'hégémonie et de déploiement de l'agencéité apparaît à la lecture de ce dossier dans le rapport à l'espace, réapproprié par les acteurs du hip-hop. Même placés sous contrainte, certains artistes parviennent à inventer des espaces de liberté renouvelés, tant dans l'espace public de la ville que par des circulations et des connexions avec des organisations transnationales.

L'article de Chloé Buire offre un premier argument pour penser les espaces de la mobilisation des rappeurs, en traitant de l'apparition d'un mouvement contre-hégémonique dans la scène rap luandaise. Cette tendance se définissant comme un rap «d'intervention sociale» surgit en Angola en réponse à la «reconversion autoritaire» des années 1990 et à l'occupation des scènes musicales par le kuduro ou par des musiques prises «dans le filet institutionnel». Chloé Buire révèle comment la création d'un contre-pouvoir s'opère par «la patiente réappropriation de sites urbains et leur transformation en véritables espaces publics, au sens fort d'agora citoyenne». De nouveaux lieux de la citoyenneté, instables mais résilients, émergent ainsi dans divers endroits stratégiques de la ville de Luanda.

Par ailleurs, l'extraversion et le recours à des connexions transnationales constituent d'autres ressorts d'émancipation bien connus, dont les rappeurs savent se saisir. Il en est ainsi pour la rappeuse tunisienne décrite par Stefano Barone et Elham Golpushnezad, qui échappe par le biais de l'exil, à une double oppression: celle du corps politique, et celle de ses homologues masculins. C'est également le cas de quelques rappeurs du Burkina Faso 
et de personnalités telles que Smockey, pilier du «Balai citoyen ${ }^{62}$ ». Leurs inscriptions dans des appartenances plurielles et multiscalaires leur permettent de s'extraire de certains mécanismes locaux de censure ou de cooptation, en forgeant des réseaux de production et d'action alternatifs ${ }^{63}$, qui réunissent une scène d'artistes ouest-africains, des associations et des ONG locales ou internationales.

Ces artistes tirent également profit des connexions tissées plus récemment avec les universitaires étrangers, démontrant le caractère itératif des rapports entre les rappeurs et le monde académique: les rappeurs ont contribué à un mouvement de la société qui a, ces vingt dernières années, retenu l'attention des observateurs, des universitaires et des journalistes. Ces derniers, avec l'appel d'air qu'ils produisent et les opportunités qu'ils provoquent en termes de mobilité, de visibilité et de financements, influent d'une manière non négligeable sur les actions et les discours des rappeurs. Y'en a marre ou le Balai Citoyen en sont des exemples éloquents: leurs leaders sont invités dans des conférences au sein d'établissements universitaires prestigieux (Sciences-Po à Paris, université de Columbia à New York, etc.) ou d'institutions internationales (Unesco, ONU, etc.), ils sont sollicités par les médias les plus reconnus et par des appels à projets qui représentent autant de débouchés lucratifs. Quoique émancipatrices et vectrices d'une nouvelle légitimité locale et internationale, ces mannes financières peuvent parfois avoir une incidence sur les alliances et les positionnements idéologiques des rappeurs.

L'exemple de la Tunisie apporte des informations éclairantes à propos des enjeux soulevés par l'implication des ONG et acteurs internationaux dans les configurations locales de la production rap. Elham Golpushnezad et Stefano Barone révèlent la volonté implicite des bailleurs de fonds internationaux de soutenir principalement des activités hip-hop conformes à l'image du rap révolutionnaire, engagé pour la transition démocratique. Ils imposent leur optique «transitologique» aux scènes locales, sans tenir compte des positionnements équivoques que doivent assumer les rappeurs ou des processus politiques en cours. Les artistes et promoteurs locaux jouent en partie le jeu imposé par ces acteurs internationaux, mais ils profitent aussi des fonds obtenus pour soutenir d'autres projets qui leur semblent plus en adéquation avec les réalités locales. Sur d'autres terrains comme au Gabon, les artistes de rap marginalisés par les scènes officielles développent eux aussi

62. Voir A. Cuomo, "Circuler pour exister: analyse de parcours de rappeurs burkinabè", communication présentée lors des troisièmes Rencontres des études africaines en France (Reaf), Bordeaux, Les Afriques dans le monde (LAM), 2014.

63. J. F. Mbaye, «AURA ou de la production politique de la musique hip-hop », Cahiers de recherche sociologique, $\mathrm{n}^{\circ} 49,2010$, p. 147-160. 
Polyphonies du rap

des rapports stratégiques avec des ONG impliquées dans la protection de l'environnement ou dans la lutte contre le sida. La réponse à leurs appels à projets et la réalisation de chansons sur ces questions sont parfois conçues comme des opportunités du marché à saisir. Grâce à ces projets, ils financent leurs entreprises musicales personnelles et trouvent une issue aux régimes verrouillés dans lesquels ils évoluent.

Au-delà d'une simple source de revenus, le secteur du développement constitue enfin un débouché professionnel dont s'emparent certains rappeurs. Ceux-ci créent leurs propres structures, s'évertuent à faire entrer leurs actions en adéquation avec les agendas des grandes organisations internationales (Unesco, etc.) ou à adopter le jargon développementaliste que celles-ci affectionnent ${ }^{64}$. Ces entrepreneurs du rap se rendent ainsi acteurs d'un soft power inversé, financé par les puissances internationales, ce qui constitue une stratégie d'extraversion, sans pour autant quitter le territoire local.

Ce dossier retrace la manière dont le rap s'est imbriqué dans les régimes politiques de différents États africains et a accompagné les dynamiques qui les traversent de l'intérieur et de l'extérieur. Sans remettre en question les ordres hégémoniques en présence ni les subvertir de l'intérieur, quelques acteurs de ces scènes hip-hop créent en leur sein des marges de manœuvre individuelles ou collectives, en reprenant selon leurs propres intérêts les règles des systèmes en présence, ou en circulant entre différents réseaux et différentes échelles.

L'un des apports de ce dossier nous semble découler de sa capacité à s'extraire des visions monolithiques et des «terrains surexploités » du rap africain $^{65}$. En révélant des scènes rap (ou des subdivisions de ces scènes rap) jusqu'à présent peu documentées, il met en évidence les modes pluriels d'imbrication et d'adaptation de ce genre musical, en fonction des situations politiques de différents États ou des mutations rencontrées au sein du même État. Par l'entrée rap, on accède alors à une compréhension plus vaste de processus de renouvellement, de transformation ou de déclin des

64. Voir le travail de L. Gaudin: «Culture et développement: quel lien l'association Africulturban, qui œuvre pour le développement des cultures urbaines au Sénégal, entretient-elle avec la notion de développement humain?», mémoire de master 2, Bordeaux, Université de Bordeaux Montaigne, 2014.

65. À propos de l'idée de terrain surexploité, voir les travaux d'A. Doquet, «L'enquête en "situation ethnologique" ou l'exercice nécessaire de la réflexivité», in L. Vidal et O. Leservoisier, L'Anthropologie face à ses objets. Nouveaux contextes ethnographiques, Paris, Archives Contemporaines, 2010, p. 205-222. On pense particulièrement au cas du mouvement Y'en a Marre, discuté par de nombreux chercheurs dans les dernières années. 
régimes concernés. Parallèlement, ce dossier souligne l'intérêt de comprendre le rap à l'intérieur d'une plus vaste scène culturelle et musicale, avec laquelle elle entretient des rapports variables en fonction des contextes, passant de la concurrence à l'intégration ou à la distinction. Enfin, il met en évidence la participation ambiguë des acteurs internationaux, du côté des ONG autant que dans les médias et le monde de la recherche, à la production du « rap africain», à la fois par les représentations fantasmées des rappeurs qu'ils véhiculent, et par les mannes financières qu'ils apportent.

En creux, ce dossier nous semble finalement et ironiquement confirmer que c'est lorsque l'on évacue la tentation de rechercher le sens politique du rap que se révèlent, de la façon la plus nuancée, les mécaniques du pouvoir des scènes rap et les processus politiques plus vastes qu'elles accompagnent.

Loin d'épuiser les discussions, ce dossier laisse en suspens plusieurs problématiques qui requerraient sûrement de faire l'objet d'examens détaillés. Ainsi, l'observation précise des acteurs du rap privilégiée ici mériterait certainement que l'on s'intéresse plus en profondeur à ses publics ou au rôle-clé des médias (radios, télé et Internet), qui constituent une scène d'une autre nature pour des rappeurs transformés en animateurs ${ }^{66}$. En outre, la présence grandissante d'un secteur culturel porté à la fois par le monde du développement et de la coopération internationale pose la question de l'éventuelle conversion des cultures urbaines en instrument efficace de la diplomatie. Enfin, les dialogues avec les acteurs de la diaspora africaine à l'étranger et les collaborations croissantes entre artistes africains et réseaux de production occidentaux pourraient très certainement faire l'objet de recherches approfondies, en lien avec les nationalismes alternatifs et avec les pratiques de l'État dans la diaspora. Le champ de recherches sur le rap en Afrique reste en somme ouvert aux métamorphoses que ce genre musical continuera de rencontrer au gré des soubresauts de l'histoire

Alice Aterianus-Owanga Laboratoire d'anthropologie et d'histoire de l'institution de la culture (LAHIC) Institut interdisciplinaire d'anthropologie du contemporain (IIAC UMR 8177)

Sophie Moulard Les Afriques dans le monde (LAM), Sciences Po Bordeaux

66. Ainsi, de nombreux rappeurs animent désormais des émissions d'actualités musicales ou des débats à la radio et à la télévision. Un autre exemple est le «Journal rappé » (JTR) au Sénégal. 